\title{
Higgs boson measurements in final states with photons at CMS
}

\author{
Hualin Mei on behalf of the CMS Collaboration* \\ University of California, Santa Barbara, United States \\ E-mail: hualin.mei@cern.ch
}

Measurements of Higgs boson properties in events where the Higgs boson decays into a pair of photons are presented. The measurements are based on a sample of proton-proton collisions at $\sqrt{s}=13 \mathrm{TeV}$ collected by the CMS detector at the LHC from 2016 to 2018, corresponding to an integrated luminosity of $137 \mathrm{fb}^{-1}$. All major Higgs production modes (gluon fusion, vector boson fusion, vector boson associated production, and production associated with top quarks) are considered in the analysis. Properties of the Higgs boson, including standard model signal strength modifiers, production cross sections, and its couplings to other particles, are found to be in agreement with the standard model expectations.

40th International Conference on High Energy physics - ICHEP2020

July 28 - August 6, 2020

Prague, Czech Republic (virtual meeting)

${ }^{*}$ Speaker 


\section{Introduction}

Since its observation the properties of the Higgs boson $(\mathrm{H})$ have been studied using a variety of decay channels and production modes. All results so far have been consistent with the corresponding SM predictions. In the SM, the $H \rightarrow \gamma \gamma$ decay has a small branching fraction of approximately $0.23 \%$ but with a clean final state topology with two well-reconstructed photons. This provides a narrow invariant mass peak which can be utilized to effectively distinguish $\mathrm{H}$ from background processes. As a consequence, $H \rightarrow \gamma \gamma$ is one of the most important channels for precision measurements of $\mathrm{H}$ properties. Moreover, it is one of the few decay channels that is sensitive to all the principal $\mathrm{H}$ production modes. The measurements reported in this note are based on data from proton-proton ( $\mathrm{pp}$ ) collisions at a center-of-mass energy of $\sqrt{s}=13 \mathrm{TeV}$ collected with the CMS detector [1] at the LHC between 2016 and 2018, corresponding to an integrated luminosity of $137 \mathrm{fb}^{-1}$. The strategy of this analysis is designed to enable measurements within the simplified template cross section (STXS) framework [2], which also allows the measurements of other $\mathrm{H}$ properties. These include standard model signal strength modifiers, production cross sections, and the H's couplings to other particles.

\section{Analysis strategy}

$\mathrm{H}$ candidates are built from pairs of photon candidates, where the narrow signal peak is distinguishable from the falling background diphoton invariant mass spectrum. Diphoton selection is performed using properties of the reconstructed diphoton system to increase the ratio of selected signal events to background events (S/B). Photon candidates are reconstructed from energy clusters in the ECAL not linked to any extrapolated charged particle trajectories. Photon energy are corrected with a multivariate regression technique to account for reconstruction effect which also predicts its uncertainty. $\mathrm{Z} \rightarrow \mathrm{e}^{+} \mathrm{e}^{-}$events are used to derive additional corrections (with electrons reconstructed as photons), including correction of drift of energy scale in data over time as a function of LHC fill, aligning dielectron mass distribution between data and simulation to correct photon energy scale (resolution) in data (simulation). Photons in events passing the preselection criteria are further required to satisfy a photon identification criterion from a boosted decision tree (BDT) trained to separate genuine ("prompt") photons from jets mimicking a photon. Simulated inputs for the photon ID BDT, both shower shape and isolation variables, are corrected to agree with data using a chained quantile regression method. Fig. 1 shows $m_{e e}$ distribution comparisons between data and simulation after all corrections are applied, as well as distribution of the photon identification BDT score of the lowest scoring photon in diphoton pairs. In both cases the distributions for the data and simulation are compatible within the uncertainties.

To enable the measurements of the cross section of each individual production mode, dedicated selection criteria and classifiers are used to select events consistent with the $\mathrm{tH}, \mathrm{ttH}, \mathrm{VH}, \mathrm{VBF}$, and ggH modes. The analysis categories targeting a given production mode is further divided to differentiate between the various STXS bins, in order to measure cross sections of STXS bins individually. For most production modes, the divisions are made using the reconstructed detector level equivalents of the particle level quantities that are used to define the bins. The analysis' sensitivity to the individual production mode and total $\mathrm{H}$ cross sections are maintained by using 

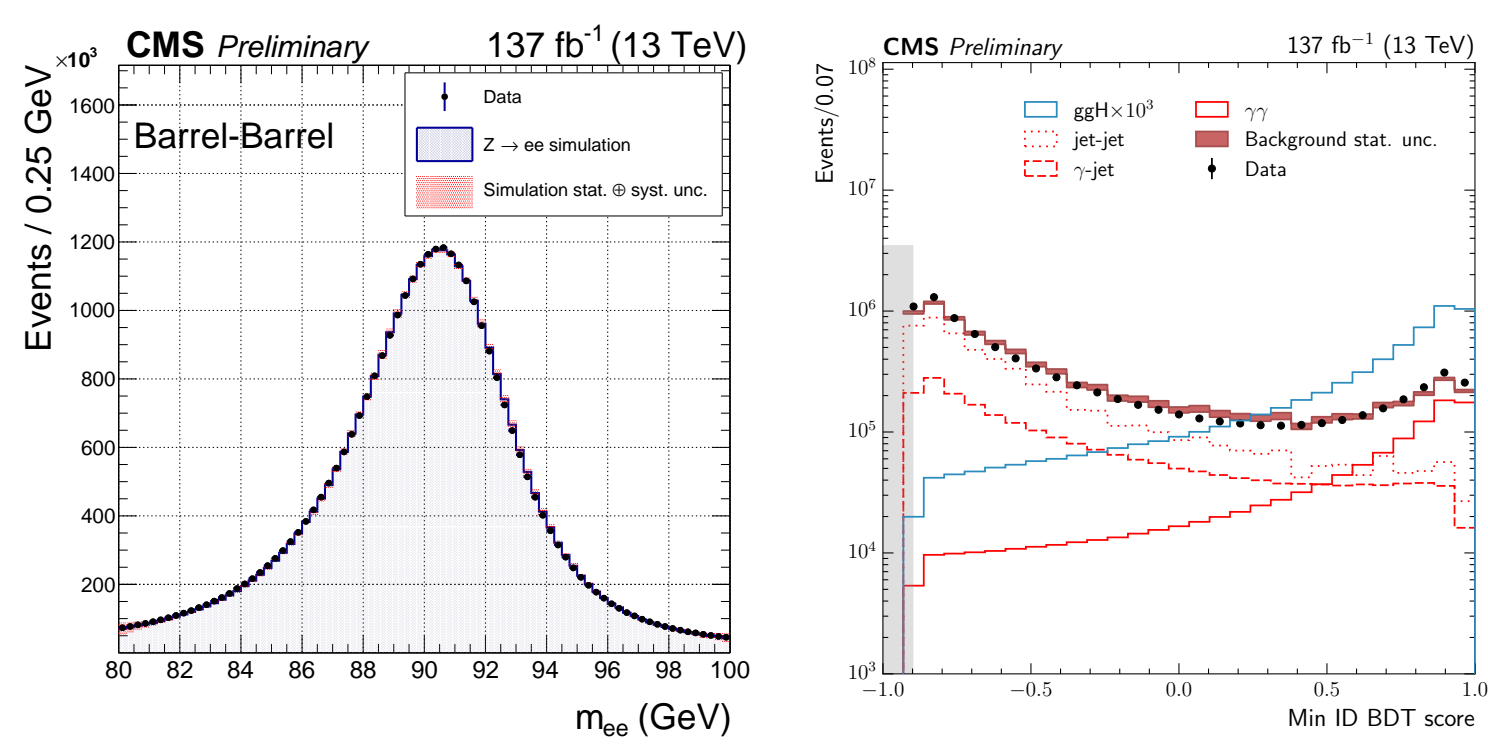

Figure 1: (Left) Comparison of the dielectron invariant mass spectra in data and simulation. (Right) Distribution of the photon identification BDT score of the lowest scoring photon in diphoton pairs with an invariant mass in the range $100<m_{\gamma \gamma}<180 \mathrm{GeV}$, for data events passing the preselection (black points), and for simulated background events. [3]

this categorization. To avoid ambiguity (an event can pass the selection criteria for more than one analysis category), a priority sequence is defined such that events could enter more than one analysis category are assigned to the category with the highest priority. Categories with a lower expected signal yield have a higher priority. Fig. 2 shows signal models for each year individually, and for the sum of the three years together as well as invariant mass distribution of the diphoton system in data from three years.

\section{Results}

A common signal strength modifier, $\mu$, is defined as the ratio of the observed product of the $\mathrm{H}$ cross section and diphoton branching fraction to the SM expectation. It is measured to be

$$
\mu=1.03_{-0.09}^{+0.11}=1.03_{-0.05}^{+0.07}(\text { theo })_{-0.03}^{+0.04}(\text { syst })_{-0.06}^{+0.07}(\text { stat }) .
$$

The uncertainty is decomposed into theoretical systematic, experimental systematic, and statistical components. The compatibility of this fit with respect to the SM prediction, expressed as a p-value, is approximately $74 \%$. Signal strength modifiers for each $\mathrm{H}$ production mode are also measured. $\mu_{\text {top }}$ scales the $\mathrm{ttH}, \mathrm{tHq}$ and $\mathrm{tHW}$ production modes equally, the $\mathrm{VH}$ hadronic and $\mathrm{VH}$ leptonic processes are grouped to scale according to $\mu_{\mathrm{VH}}$, the VBF production mode scales with $\mu_{\mathrm{VBF}}$ and $\mu_{\mathrm{ggH}}$ scales both ggH and bbH production. The values of the production mode signal strength modifiers and their uncertainties are shown in Fig. 3. The compatibility of this fit with respect to the SM prediction is approximately $53 \%$.

Two different fits to the cross sections of partially merged STXS bins are presented in this analysis. The first is referred to as the "maximal" merging scenario, where in general STXS 

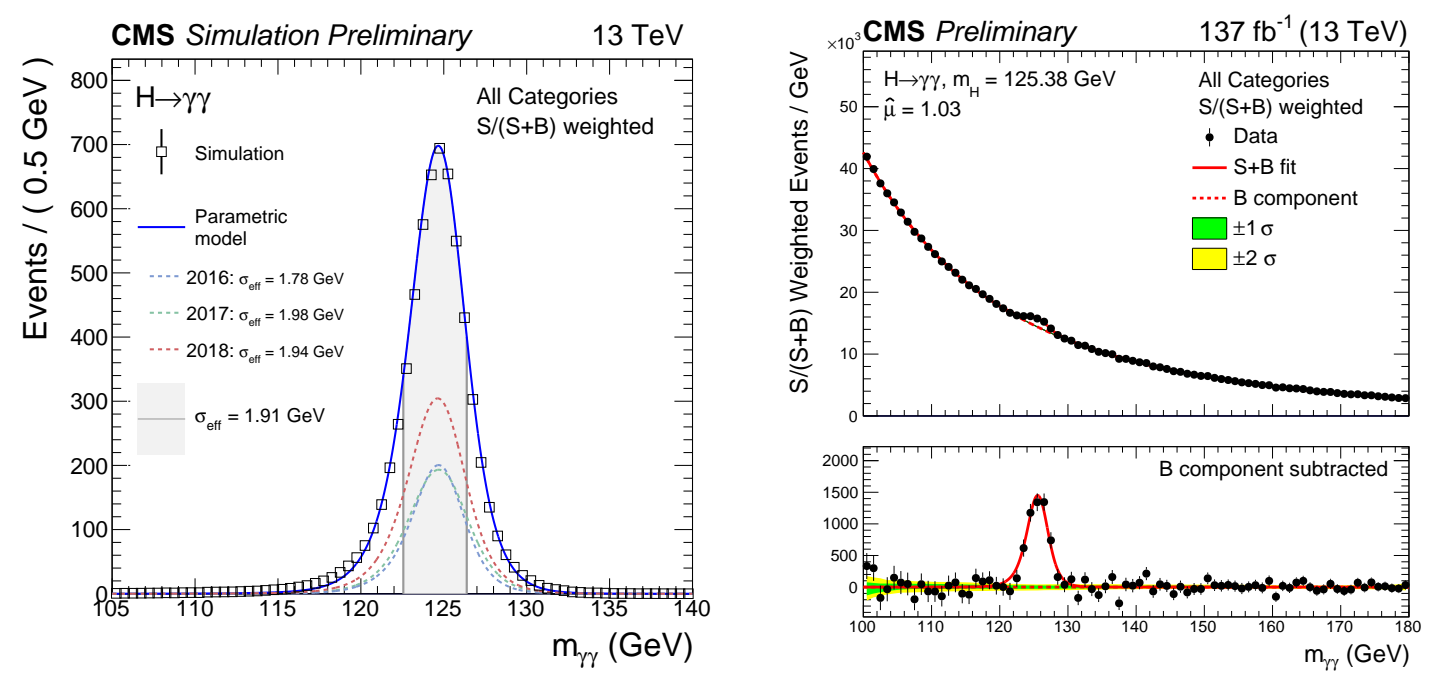

Figure 2: (Left) The shape of the parametric signal model for each year of simulated data, and for the sum of all years together, is shown. The models are shown for the weighted sum of all categories. Here each category is weighted by $\mathrm{S} /(\mathrm{S}+\mathrm{B})$, where $\mathrm{S}$ and $\mathrm{B}$ are the numbers of expected signal and background events, respectively, in a $\pm 1 \sigma_{\text {eff }}$ mass window centered on $\mathrm{m}_{\mathrm{H}}$. (Right) Data points (black) and signalplus-background model fit for the sum of all categories is shown. Each category is weighted by $\mathrm{S} /(\mathrm{S}+$ B). [3]

bins are merged until their expected uncertainty is less than $150 \%$ of the SM prediction. The maximal merging scheme defines 17 parameters of interest. The second "minimal" merging fit instead merges as few bins as possible and ensuring that correlations between parameters remain smaller than approximately 0.75 . The minimal merging scheme defines a more granular fit with 24 parameters of interest. The best fit cross sections and $68 \%$ confidence level intervals are shown for the minimal merging schemes in Figs. 4. The same fit result for maximal merging scheme and correlations between the fitted parameters can be found in [3], which also has detailed discussion of this analysis. Both the maximal and minimal fits are dominated by the statistical component of the uncertainty for all measured cross sections. All results are in agreement with SM predictions.

The $\kappa$-framework [4] defines coupling modifiers to directly parametrise deviations from the SM expectation in the couplings of the $\mathrm{H}$ to other particles. Two different two dimensional likelihood scans are performed. The first fit uses the resolved $\kappa$ model is used. Here the scaling factors of the $\mathrm{ggH}$ and $H \rightarrow \gamma \gamma$ loops are resolved into their SM components, in terms of the other $\kappa$ parameters. The fitted results of a two-dimensional scan in $\kappa_{\mathrm{V}}$ and $\kappa_{\mathrm{f}}$, each scales the H's coupling to vector bosons and to fermions, respectively, are shown in the left plot of Fig. 5. In the scan, the other parameters are fixed to unity. This analysis explicitly targets tHq production via the tHq leptonic analysis category, the inclusion of this category in the fit reduces the degeneracy between positive and negative $\kappa_{\mathrm{f}}$ values. The region with negative values of $\kappa_{\mathrm{f}}$ is observed (expected) to be excluded with a significance of $1.0(2.3)$. A second fit is performed using the unresolved $\kappa$ model, where the $\mathrm{ggH}$ and $H \rightarrow \gamma \gamma$ loops are given their own effective scaling factors denoted as $\kappa_{\mathrm{g}}$ and $\kappa_{\gamma}$, respectively. The observed results of a two-dimensional scan in these two parameters are shown in the right plot of Fig. 5. In the scan, the other $\kappa$ parameters in the unresolved model are fixed to 
unity.

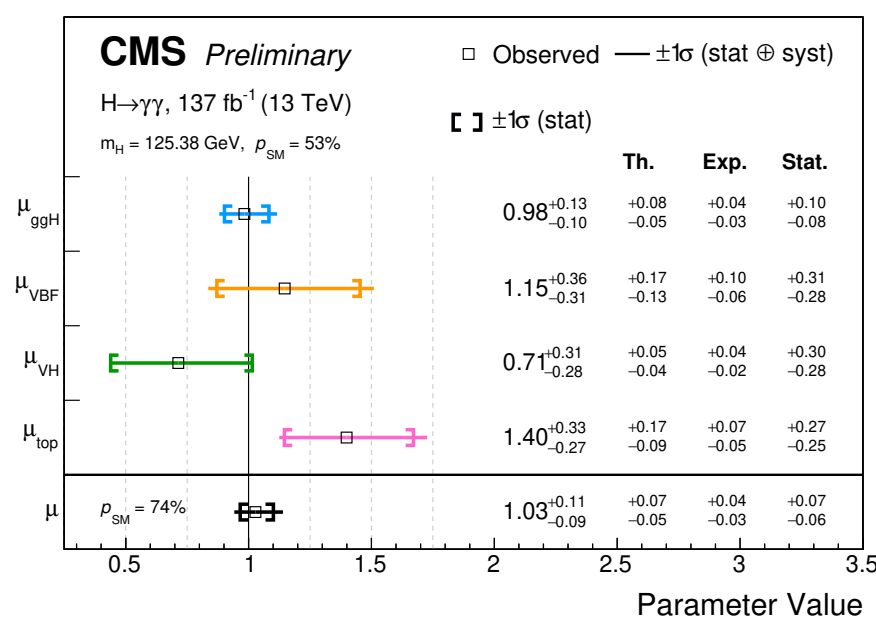

Figure 3: Observed results of the fit to four production mode signal strength modifiers. The contributions to the total uncertainty in each parameter from the theoretical systematic, experimental systematic and statistical components are shown. Also shown in black is the result of the fit to the inclusive signal strength modifier. The compatibility of this fit with respect to the SM prediction, expressed as a $p$-value, is approximately $53 \%$. [3]

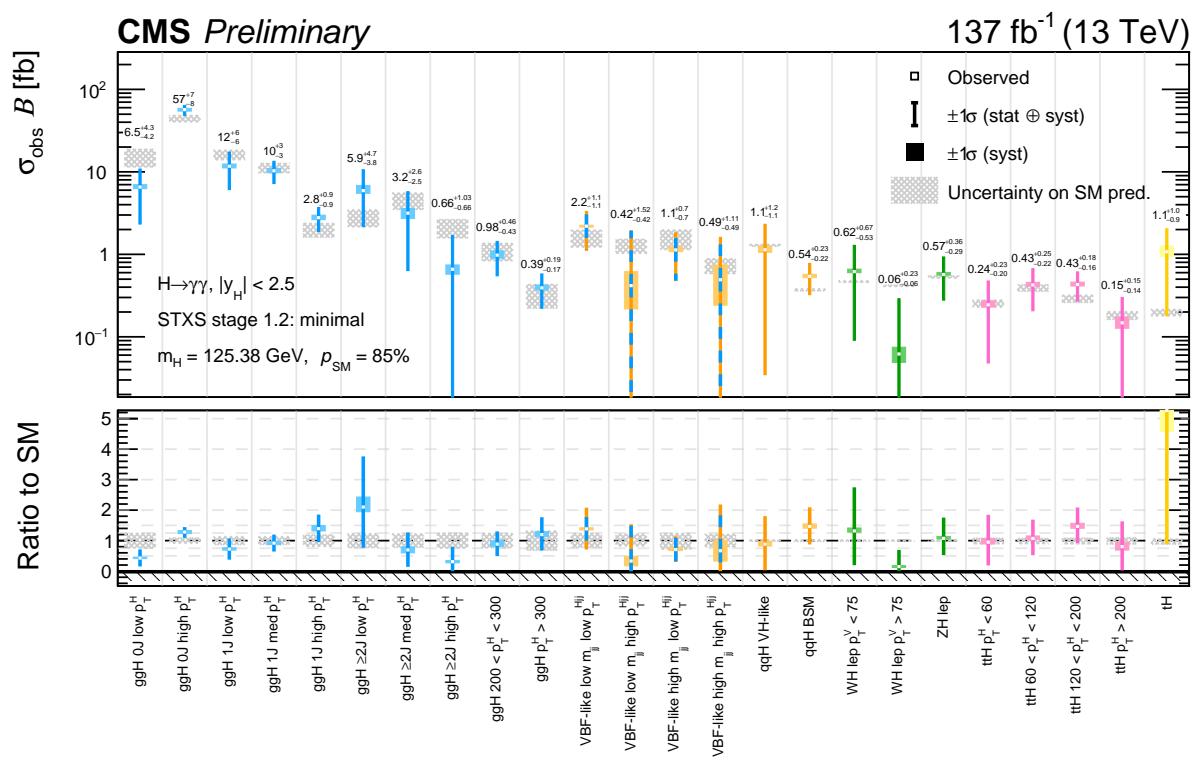

Figure 4: Observed results of the minimal merging scheme STXS fit. The best fit cross sections are plotted along with the respective $68 \%$ confidence level intervals. The systematic components of the uncertainty in each parameter are shown by the coloured boxes. The hatched grey boxes demonstrate the theoretical uncertainties in the SM predictions. The bottom panel shows the ratio of the fitted values to the SM predictions. The orange and blue dashed lines for the VBF-like parameters represents contributions from both ggH and qqH STXS bins. The compatibility of this fit with respect to the SM prediction, expressed as a $p$-value, is approximately $85 \%$. [3] 

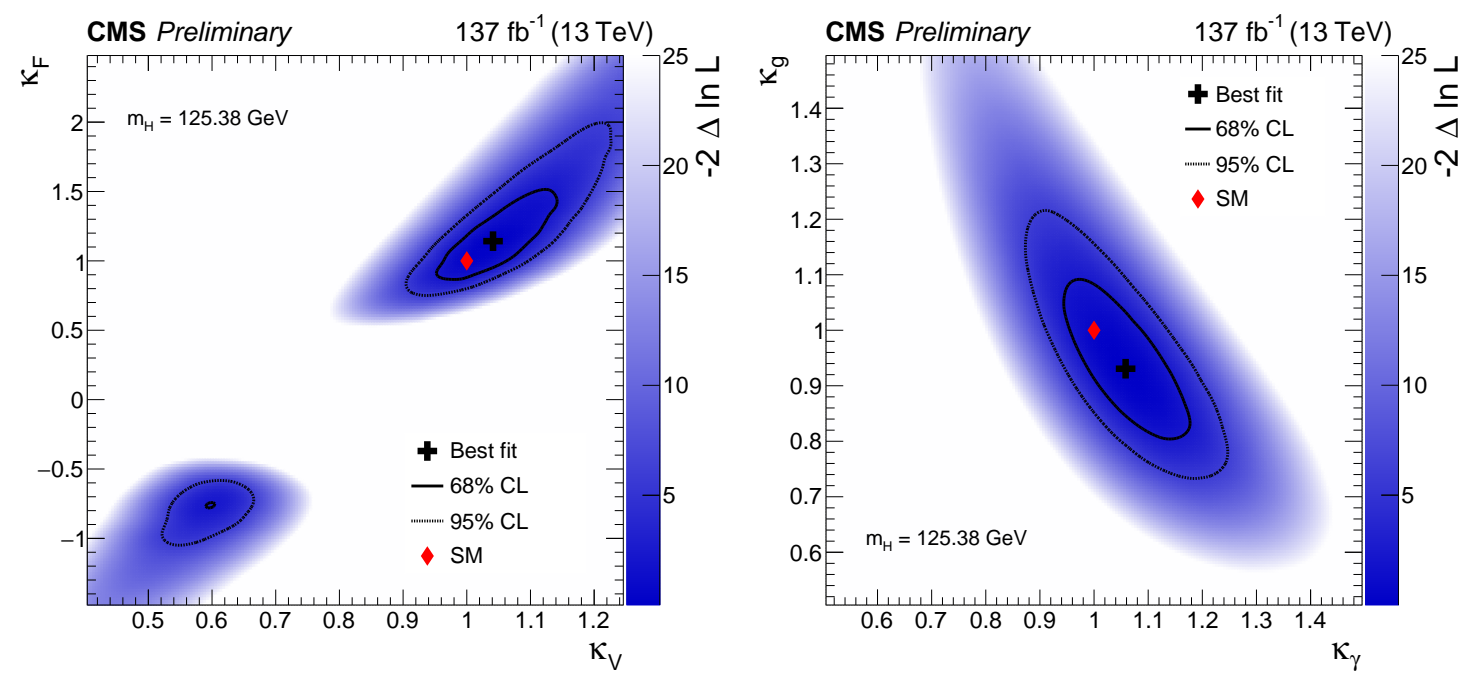

Figure 5: Observed two dimensional likelihood scans performed in the $\kappa$-framework: $\kappa_{\mathrm{V}}$ versus $\kappa_{\mathrm{f}}$ in the resolved $\kappa$ model (left) and $\kappa_{\gamma}$ versus $\kappa_{\mathrm{g}}$ in the unresolved $\kappa$ model (right). The $68 \%$ and $95 \%$ confidence level regions are given by the solid and dashed contours, respectively. [3]

\section{Conclusion}

Recent measurements of $\mathrm{H}$ properties with the $\mathrm{H}$ decaying into a pair of photons are discussed in this report. Events with two photons are selected from a sample of proton-proton collisions at a center-of-mass energy $\sqrt{s}=13 \mathrm{TeV}$ collected by the CMS detector at the LHC from 2016 to 2018, corresponding to an integrated luminosity of $137 \mathrm{fb}^{-1}$. The signal strength modifiers, production cross sections, and its couplings to other particles are measured, all results are found to be consistent with the standard model expectations.

\section{References}

[1] CMS Collaboration, The CMS experiment at the CERN LHC, JINST 3 (2008) S08004.

[2] LHC Higgs Cross Section Working Group, Handbook of LHC Higgs cross sections: 4. deciphering the nature of the Higgs sector, CERN (2016) [1610.07922].

[3] CMS Collaboration, Measurements of Higgs boson properties in the diphoton decay channel at $\sqrt{s}=13 \mathrm{TeV}$, Tech. Rep. CMS-PAS-HIG-19-015, CERN, Geneva (2020).

[4] LHC Higgs Cross Section Working Group, Handbook of LHC Higgs cross sections: 3. Higgs properties, CERN (2013) [1307.1347]. 